\title{
Cytokine expression patterns and mesenchymal stem cell karyotypes from the bone marrow microenvironment of patients with myelodysplastic syndromes
}

\author{
H. Xiong*, X.Y. Yang*, J. Han, Q. Wang and Z.L. Zou \\ Department of Hematology, Shanghai Clinical Research Center, Chinese Academy of Sciences, \\ Shanghai Xuhui District Central Hospital, Shanghai, China
}

\begin{abstract}
The purpose of this study was to explore cytokine expression patterns and cytogenetic abnormalities of mesenchymal stem cells (MSCs) from the bone marrow microenvironment of Chinese patients with myelodysplastic syndromes (MDS). Bone marrow samples were obtained from 30 cases of MDS (MDS group) and 30 healthy donors (control group). The expression pattern of cytokines was detected by customized protein array. The karyotypes of MSCs were analyzed using fluorescence in situ hybridization. Compared with the control group, leukemia inhibitory factor, stem cell factor (SCF), stromal cell-derived factor (SDF-1), bone morphogenetic protein 4, hematopoietic stem cell (HSC) stimulating factor, and transforming growth factor- $\beta$ in the MDS group were significantly downregulated $(\mathrm{P}<0.05)$, while interferon- $\gamma($ IFN- $\gamma)$, tumor necrosis factor- $\alpha$ (TNF$\alpha$ ), and programmed death ligand $(\mathrm{B} 7-\mathrm{H} 1)$ were significantly upregulated $(\mathrm{P}<0.05)$. For chromosome abnormality analysis, the detection rate of abnormal karyotypes $(+8,-8,-20,20 q-,-Y,-7,5 q-)$ was $30 \%$ in the MDS group and $0 \%$ in the control group. In conclusion, the up- and downregulated expression of these cytokines might play a key role in the pathogenesis of MDS. Among them, SCF and SDF-1 may play roles in the apoptosis of HSCs in MDS; and IFN- $\gamma$, TNF- $\alpha$, and B7-H1 may be associated with apoptosis of bone marrow cells in MDS. In addition, the abnormal karyotypes might be actively involved in the pathogenesis of MDS. Further studies are required to determine the role of abnormal karyotypes in the occurrence and development of MDS.
\end{abstract}

Key words: Bone marrow microenvironment; Cytokine; Mesenchymal stem cell; Chromosome; Pathogenesis

\section{Introduction}

Myelodysplastic syndromes (MDS) are a group of diseases derived from malignant amplification of hematopoietic stem cells (HSCs), with typical features of abnormal myeloid cell differentiation and maturation, ineffective hematopoiesis, and a high risk of progression to acute myeloid leukemia (1). The incidence and prevalence of MDS are higher in males than in females and increase sharply with age $(2,3)$. With the aging population and improving awareness of the disease, the documented disease burden is predicted to escalate in the near future. Moreover, the age of onset is younger in Asia than in Western countries, $(4,5)$. Therefore, there is an urgent need to develop new therapeutic options and clinical management of MDS, which must be based on diagnostic efficiency and knowledge of the pathology of MDS.
Currently, the diagnosis of MDS is complex (6), because signs and symptoms for MDS are nonspecific and are generally related to blood cytopenias $(7,8)$. MDS must be distinguished from anemia, thrombocytopenia, and leukopenia $(9,10)$. Hence, it is important to find specific pathological hallmarks for improving diagnostic efficiency.

Based on previous studies, MDS are characterized by complicated pathological hallmarks such as abnormal HSCs (11), changes in the hematopoietic microenvironment (12), and immune system defects (13). As the niche for HSCs, the bone marrow hematopoietic microenvironment is a complicated network system containing the bone marrow microcirculation and mesenchymal stromal cellular components and cytokines (14). These cytokines are important for the growth and survival of HSCs $(15,16)$

Correspondence: Hong Xiong: <hxiong@scrc.ac.cn>.

${ }^{*}$ These authors contributed equally to this study.

Received May 8, 2014. Accepted September 2, 2014. First published online January 20, 2015. 
and antagonistically or synergistically induce excessive apoptosis of HSCs during the occurrence and development of MDS (17). However, the specific roles of these cytokines are still unclear. In addition, an abnormal karyotype is the key genetic feature of MDS pathogenesis. Although cytogenetic abnormalities of MSCs in MDS have been observed in some previous studies (18-22), it is still necessary to further investigate the karyotypic features of Chinese MDS patients, because there are population differences in genetic makeup, especially between Asian and Western peoples $(5,22)$. We, therefore, studied 30 Chinese patients with MDS and observed the expression pattern of cytokines and abnormal karyotypes of MSCs in their bone marrow microenvironment.

\section{Patients and Methods}

All patients gave their written informed consent prior to inclusion in this study, which was approved by the Clinical Research Center, Chinese Academy of Sciences, Shanghai Xuhui District Central Hospital Ethics Committee, and performed in accordance with their ethical standards.

\section{Patients}

A total of 30 Chinese patients with presumptively diagnosed MDS (15 males and 15 females, age ranging from 40 to 68 years with a median of 50 years) were evaluated in this study. According to World Health Organization criteria (23), the patients were classified as follows: 8 cases of refractory anemia, 7 cases of refractory anemia with ringed sideroblasts, 7 cases of refractory anemia with excess blasts, and 8 cases of refractory anemia with excess blasts in transformation. None of the patients were diagnosed as having had other diseases, except myelodysplastic syndromes, previously. Moreover, none of them had received either chemotherapy or immunosuppressant treatments.

In addition, 30 healthy donors (15 males and 15 females, age ranging from 36 to 66 years with a median of 49 years) were included as normal controls.

\section{Bone marrow samples}

Bone marrow samples were derived from 30 MDS patients (MDS group) and 30 healthy donors (control group). Bone marrow aspiration and biopsy were performed under conscious sedation with intravenous propofol and local infiltration with lidocaine. Bone marrow aspirates were obtained from the posterior superior iliac crest and collected into the alpha medium containing $10 \mathrm{U} / \mathrm{mL}$ preservative-free heparin. Then the mononuclear cells (MNCs) were isolated by Ficoll density gradient centrifugation (GE Healthcare, USA), the supernatant was used for protein array hybridization, and the isolated MNCs were collected and cultured.

\section{Protein array preparation and hybridization}

The supernatant of bone marrow was used for protein array hybridization with a customized cytokine antibody array from RayBio Human Cytokine Antibody Array (RayBiotech, Inc., USA) according to standard operational protocols. The protein array map is shown in Table 1. Pretest results showed that there was no cross-reaction among the cytokine antibodies. Chemiluminescent signals were detected using a Fuji LAS1000 imaging system (Fujifilm, Japan) and then analyzed using the Advanced Image Data Analyzer software (AIDA, 3.28; Raytest Izotopenmessgeraete, Germany). Expression levels of cytokines are reported as signal intensity values.

\section{Cell cultures}

Isolated MNCs from the MDS group and the control group were cultured in MesenCult MSC basal medium (Human, Stem Cell Technologies, Canada) at $37^{\circ} \mathrm{C}$ in a humidified incubator with $5 \% \mathrm{CO}_{2}$ in air. The cells were subcultured to passage three (P3), and the P3 cells were then obtained by trypsin digestion and centrifugation at $1082 \mathrm{~g}$ for $5 \mathrm{~min}$.

\section{Flow cytometry analysis}

Flow cytometric analysis was performed to confirm the types of cells using specific surface antigens of MSCs (CD29 and CD90) and HSCs (CD34 and CD45). The cells in culture were trypsinized and stained with fluorescein isothiocyanate (FITC) or phycoerythrin (PE) conjugated antibodies, including FITC-CD34, PE-CD90, FITC-CD45, and PE-CD29 monoclonal antibodies (Immunotech, USA). Ten thousand labeled cells were acquired and analyzed using a Becton Dickinson flow cytometer (USA). Data (collected in list mode) were analyzed using the EXPO32 ADC software (Beckman Coulter, Germany).

\section{Fluorescence in situ hybridization (FISH) detection}

The P3 bone marrow MSCs were collected for interphase FISH analysis using a FISH kit (Beijing GP Medical Technologies, China). The following probes were used for hybridization: LSI EGR1 (5q31)/D5S721, D5S23 (5p15.2), LSI D7S486 (7q31)/CEP 7 SG (CSP7), LSI D20S108 (20q12)/CSP8, and CSPX/CSPY (Beijing GP Medical Technologies). Procedures were performed according to standard Abbott/Vysis protocols. Briefly, the cells were collected by centrifugation at $128,778 \mathrm{~g}$ for $10 \mathrm{~min}$. The cell pellets were gently mixed with a small volume of paraformaldehyde, and then $5 \mu \mathrm{L}$ of the cell mixtures was placed on the slide and dried naturally. The slides were then fixed in an oven at $56^{\circ} \mathrm{C}$ for $20 \mathrm{~min}$, and afterwards soaked consecutively in $100 \%$ methanol for $5 \mathrm{~min}, 100 \%$ ethanol for $30 \mathrm{~min}, 70 \%$ ethanol for $2 \mathrm{~min}$, $85 \%$ ethanol for $2 \mathrm{~min}$, and $100 \%$ ethanol for $2 \mathrm{~min}$. After they were soaked, the slides were dried naturally and heated to $56^{\circ} \mathrm{C}$ again. Then, $10 \mu \mathrm{L}$ of the probe mixtures was added on the surface of the slides and immediately 
Table 1. Map of the protein array.

\begin{tabular}{cccccccccc}
\hline & A & B & C & D & E & F & G & H & I \\
\hline 1 & Pos1 & Pos2 & Pos3 & Neg & LIF & SCF & EGF & CCL2 & SDF-1 \\
2 & Pos1 & Pos2 & Pos3 & Neg & LIF & SCF & EGF & CCL2 & SDF-1 \\
3 & BMP-4 & IL-2 & IFN- $\gamma$ & Neg & TNF- $\alpha$ & CXCR4 & FLT-3 & MIG & TGF- $\beta$ \\
4 & BMP-4 & IL-2 & IFN- $\gamma$ & Neg & TNF- $\alpha$ & CXCR4 & FLT-3 & MIG & TGF- $\beta$ \\
5 & XIAP & Notch1 & IGF-I & IL-8 & B7-H1 & Neg & ANG & Neg & IL-1 \\
6 & XIAP & Notch1 & IGF-I & IL-8 & B7-H1 & Neg & ANG & Neg & IL-1 \\
\hline
\end{tabular}

LIF: leukemia inhibitory factor; SCF: stem cell factor; SDF-1: stromal cell-derived factor; BMP-4: bone morphogenetic protein 4; FLT-3: HSC-stimulating factor; TGF- $\beta$ : transforming growth factor- $\beta$; IFN- $\gamma$ : interferon- $\gamma$; TNF- $\alpha$ : tumor necrosis factor- $\alpha$; B7-H1: programmed death ligand; Pos: photoreceptor outer segment proteins; Neg: endo-1,6-beta-D-glucanase; EGF: epidermal growth factor; CCL2: monocyte chemoattractant protein 1; CXCR4: CXC chemokine receptor-4; IL-2: interleukin-2; MIG: membrane immunoglobulin; XIAP: X-linked inhibitor of apoptosis; Notch1: neurogenic locus notch homolog protein 1.

covered with a cover glass. Hybridization was performed on the hybridization apparatus for $16 \mathrm{~h}$. After they were washed, the slides were dried naturally in darkness. The slides were then counterstained with $15 \mu \mathrm{L}$ of the fluorescent dye 4',6-diamidino-2-phenylindole $2 \mathrm{HCl}$ in darkness for 20 min and observed under a fluorescence microscope (Olympus BX 50, Japan). In total, 200 HSC nuclei and 500 MSC nuclei were scored.

\section{Statistical analysis}

All data were analyzed with the SPSS 11.0 software. Statistical differences were determined using Student's $t$-test for independent samples. A $P$ value $<0.05$ was considered significant.

\section{Results}

\section{Expression patterns of cytokines in the bone marrow} microenvironment

Compared to the control group, there were significantly lower levels of leukemia inhibitory factor (LIF), stem cell factor (SCF), stromal cell-derived factor (SDF-1), bone morphogenetic protein 4 (BMP-4), HSC-stimulating factor (FLT-3), and transforming growth factor- $\beta$ (TGF- $\beta$; $\mathrm{P}<0.05)$ and higher levels of interferon- $\gamma($ IFN- $\gamma)$, tumor necrosis factor- $\alpha$ (TNF- $\alpha$ ), and programmed death ligand $(\mathrm{B} 7-\mathrm{H} 1 ; \mathrm{P}<0.05)$ in the MDS group (Figure 1).

\section{Immune phenotypic analysis}

The expression levels of cell surface markers, including bone marrow MSC surface markers (CD29 and CD90), HSC surface marker CD34, and leukocyte surface marker CD45, were detected using a flow cytometer. The results indicated that the HSC surface marker CD34 and leukocyte surface marker CD45 were not expressed in the MDS group nor in the control group. Meanwhile, the MSC markers (CD29 and CD90) were expressed in both the MDS and the control groups. Our results suggested that the cells isolated from bone marrow in MDS and control groups were confirmed as MSCs (Figure 2).

\section{Chromosome abnormality analysis}

As expected, no chromosomal abnormalities were detected in MSCs derived from the 30 healthy donors in the control group. However, 9 of the 30 MDS patients (abnormal karyotype rate of $30 \%$ ) showed abnormal karyotypes ( $+8,-20,-8,20 q-,-7,-Y, 5 q-)$ in MSCs (Figure 3, Table 2).

\section{Discussion}

Cytokines in the bone marrow microenvironment have been reported to play an important role in supporting the growth and survival of HSCs (24). In this study, we found that the expression levels of cytokines including LIF, SCF, SDF-1, BMP-4, FLT-3, and TGF- $\beta$ were significantly downregulated in the bone marrow microenvironment of MDS patients. Among these cytokines, SCF is the key in
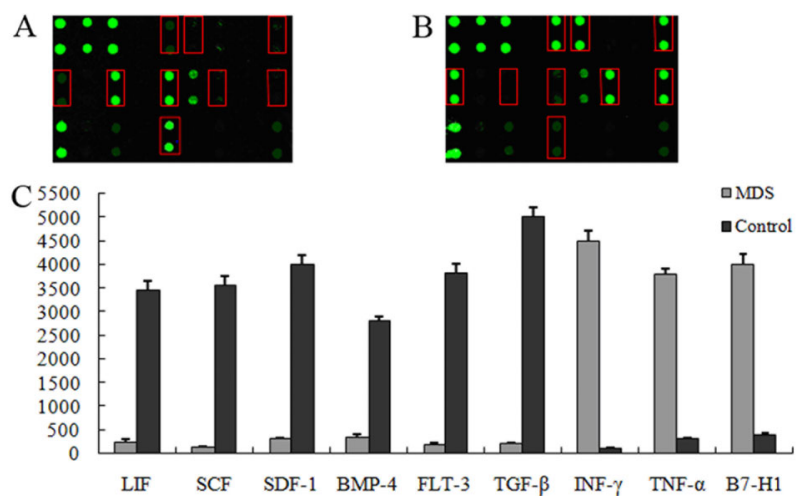

Figure 1. Expression analysis of cytokines through protein array hybridization (Student $t$-test). $A$, Signal map of myelodysplastic syndromes (MDS) group. B, Signal map of normal control group. $C$, Quantization of the expression level of cytokines by histogram plotting. LIF: leukemia inhibitory factor; SCF: stem cell factor; SDF-1: stromal cell-derived factor; BMP-4: bone morphogenetic protein 4; FLT-3: HSC-stimulating factor; TGF- $\beta$ : transforming growth factor- $\beta$; IFN- $\gamma$ : interferon- $\gamma$; TNF- $\alpha$ : tumor necrosis factor$\alpha ; \mathrm{B} 7-\mathrm{H} 1$ : programmed death ligand. 

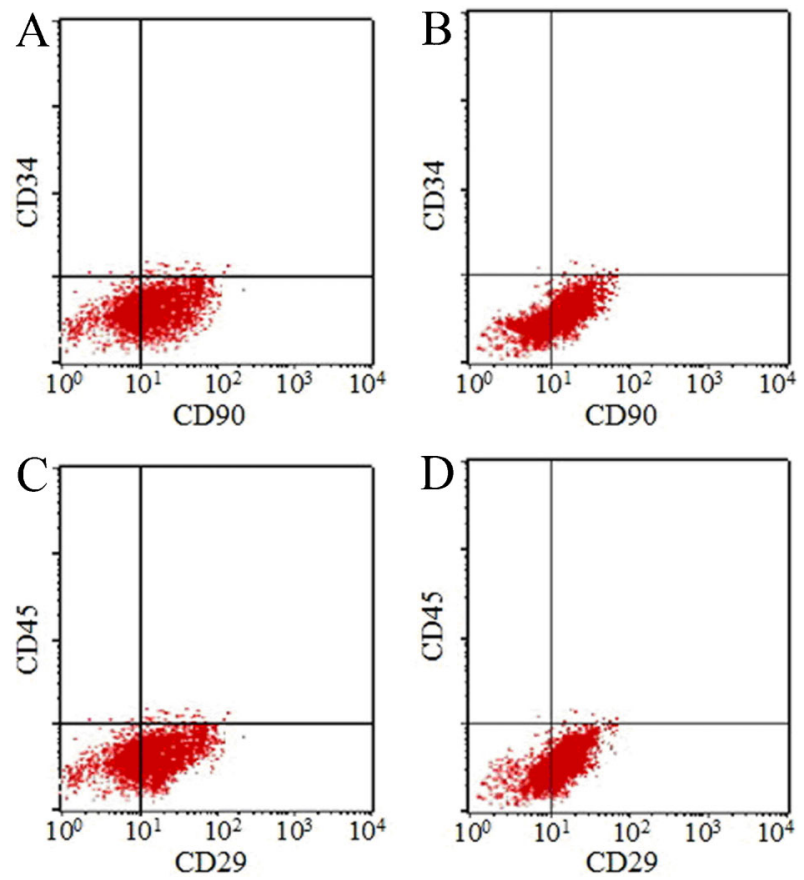

Figure 2. Immune-phenotypic analysis through flow cytometry detection. $A, C$, myelodysplastic syndromes (MDS) group; $B, D$, normal control group.

survival, proliferation, and differentiation of early HSCs $(25,26)$. Meanwhile, SCF was associated with the apoptosis of expanded HSCs. Also, as the specific receptor of CXCR4, SDF-1 is secreted by the stromal cells in bone marrow (27). Normally, SDF-1 and CXCR4 are involved in the regulation of migration, survival, and development of HSCs (28). In MDS, it has been reported that the SDF-1/CXCR4 axis and its correlation were associated with the angiogenesis and apoptosis of HSCs (29). Therefore, we inferred that SCF and SDF-1 may play roles in the apoptosis of HSCs in MDS, and that there may be some correlation between them. In addition, upregulated expression of IFN- $\gamma$, TNF- $\alpha$, and $\mathrm{B} 7-\mathrm{H} 1$ was found in MDS patients in this study, which indicated that they may play some role in the occurrence and development of MDS. Kondo et al. (30) reported that the expression of B7$\mathrm{H} 1$ was enhanced after adding IFN- $\gamma$ and TNF- $\alpha$ to the MDS cell culture medium. Cachaço et al. (31) discovered that TNF- $\alpha$ could induce apoptosis of bone marrow cells in vitro. Thus, we speculated that there may be some correction among the three cytokines for the apoptosis of bone marrow cells in MDS. In summary, the up- and downregulated expressions of these cytokines might play a key role in the pathogenesis of MDS, especially the apoptosis of cells.

The bone marrow microenvironment is formed mainly of MSCs, which can support hematopoiesis and differentiate along multiple mesenchymal lineages (32). In the chromosome abnormality analysis of this study, we found that $30 \%$ of the 30 MDS patients displayed abnormal karyotypes for MSCs, which may be a genetic marker of MSCs in MDS. However, the rate of cytogenetic aberrations in MSCs was $44 \%$ in the study by Blau et al. (20) and $64 \%$ in the study by Song et al. (18). Meanwhile, there were some differences
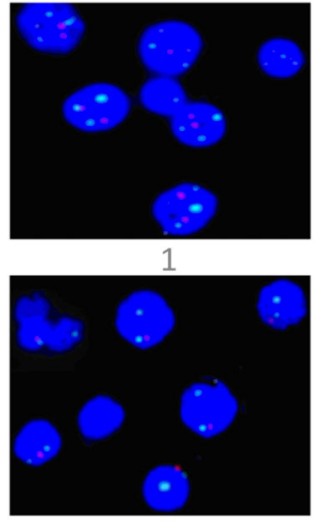

4

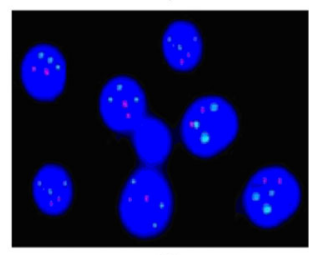

7

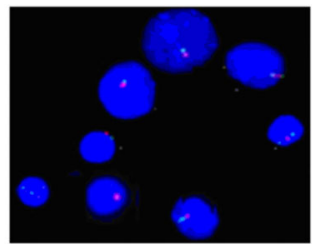

2

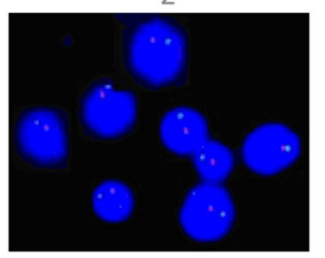

5

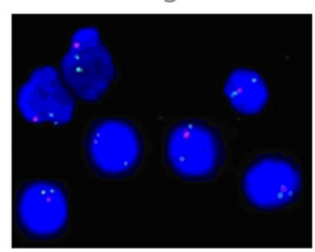

8

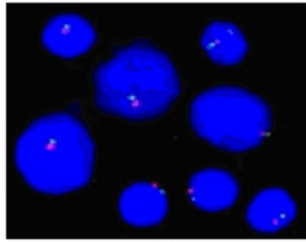

3

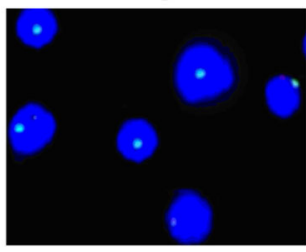

6

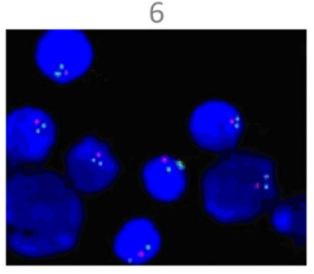

9

Figure 3. Karyotypic analysis by FISH detection. Various probes were designed for abnormal karyotype detection. 1 and 7 , karyotype of trisomy $(+8) ; 2$, deletion of chromosome 20 $(-20) ; 3$, deletion of chromosome $8(-8) ; 4$ and 8 , deletion of long arm in chromosome 20 (20q-); 5 , in situ fluorescent signal pattern for the deletion of chromosome $7(-7)$; 6 , deletion of $Y$ chromosome $(-Y) ; 9$, deletion of long arm in chromosome 5 (5q-). 
Table 2. Abnormal karyotype of mesenchymal stem cells (MSCs) from the myelodysplastic syndromes group.

\begin{tabular}{lcccc}
\hline No. & Gender & Age & MDS type & Karyotype \\
\hline 1 & Male & 48 & RA & +8 \\
2 & Male & 53 & RARS & -20 \\
3 & Female & 65 & RAEB & -8 \\
4 & Male & 60 & RAEB & $20 \mathrm{q}-$ \\
5 & Female & 63 & RAEB-T & -7 \\
6 & Male & 45 & RA & $-Y$ \\
7 & Male & 55 & RAEB & +8 \\
8 & Female & 40 & RAEB-T & $20 \mathrm{q}-$ \\
9 & Male & 42 & RARS & $5 \mathrm{q}-$ \\
\hline
\end{tabular}

MDS: myelodysplastic syndromes; RA: refractory anemia; RARS: refractory anemia with ringed sideroblasts; RAEB refractory anemia with excess blasts; RAEB-T: refractory anemia with excess blasts in transformation.

between the chromosome abnormalities $(+8,-8,-20$, $20 \mathrm{q}-,-\mathrm{Y},-7,5 \mathrm{q}-)$ in this study and those of previous studies $(18-20,33)$. Among them, the deletion of chromosome $8(-8)$ was not found in the studies by Blau et al. $(20,33)$ and Pinheiro et al. $(34)$, but it was identified in the studies by Flores-Figueroa et al. $(12,19)$ and our study. These inconsistent results may be attributed to the different detection methods (Song et al. (18), Flores-Figueroa et al. $(12,19)$, and Pinheiro et al. (34): trypsine-giemsa banding) or to the different populations (Flores-Figueroa et al. $(12,19)$ : Mexico; Blau et al. $(20,33)$ : Germany; Pinheiro et al. (34): Brazil). Thus, it is necessary to explore different karyotype abnormalities in different populations, and more studies are needed to verify the syndrome of deletion of chromosome $8(-8)$ in MDS. For the diagnosis of MDS, different karyotype abnormalities among ethnicities should be considered. In addition, deletion of the long arm of

\section{References}

1. Strom SS, Velez-Bravo V, Estey EH. Epidemiology of myelodysplastic syndromes. Semin Hematol 2008; 45: 813, doi: 10.1053/j.seminhematol.2007.10.003.

2. Neukirchen J, Schoonen WM, Strupp C, Gattermann N, Aul $\mathrm{C}$, Haas $\mathrm{R}$, et al. Incidence and prevalence of myelodysplastic syndromes: data from the Dusseldorf MDS-registry. Leuk Res 2011; 35: 1591-1596, doi: 10.1016/j.leukres. 2011.06.001.

3. Bammer C, Sperr WR, Kemmler G, Wimazal F, Nosslinger $\mathrm{T}$, Schonmetzler A, et al. Clustering of comorbidities is related to age and sex and impacts clinical outcome in myelodysplastic syndromes. J Geriatr Oncol 2014; 5: 299306, doi: 10.1016/j.jgo.2014.02.002.

4. Wang $W$, Wang $H$, Wang $X Q$, Lin GW. First report of incidence of adult myelodysplastic syndrome in China. Ann Hematol 2012; 91: 1321-1322, doi: 10.1007/s00277-011 $-1389-7$.

5. Chen B, Zhao WL, Jin J, Xue YQ, Cheng X, Chen XT, et al. chromosome 5 (5q-) was found both in our study and in studies by Blau et al. $(20,33)$. It has been reported that the MDS patients with syndrome $5 q$ - showed a specific genetic profile, which could play an important role in the pathogenesis and disease characteristics (35). Therefore, these chromosome abnormalities may be associated with the pathogenesis of MDS. Further studies are required to determine the roles of abnormal karyotypes in the occurrence and development of MDS.

In addition, in this study we only aimed to explore cytogenetic abnormalities of MSCs in MDS, so total bone marrow karyotyping was not performed. Further studies are required to investigate whether the karyotype abnormalities of HSCs and MSCs exist in MDS. Furthermore, since FISH analysis occurred in MSC cells during the third passage, contamination with clonal HSCs could be not possible in this study.

In conclusion, nine potentially key cytokines (downregulated cytokines: LIF, SCF, SDF-1, BMP-4, FLT-3, and TGF- $\beta$; upregulated cytokines: IFN- $\gamma$, TNF- $\alpha$, and B7-H1) in MDS pathogenesis were found. Among them, SCF and SDF-1 may play roles in the apoptosis of HSCs in MDS; and IFN- $\gamma$, TNF- $\alpha$ and $B 7-\mathrm{H} 1$ may be associated with the apoptosis of bone marrow cells in MDS. Abnormal karyotypes $(+8,-8,-20,20 q-,-Y,-7,5 q-)$ in MSCs were detected in $30 \%$ of Chinese MDS patients. These results indicated that these abnormal karyotypes might be actively involved in the pathogenesis of MDS. Furthermore, the up- and downregulation of cytokines as well as abnormal karyotypes could serve as indicators for MDS diagnosis and prognosis.

\section{Acknowledgments}

Research supported by Shanghai Municipal Natural Science Foundation (\#13ZR1438800).
Clinical and cytogenetic features of 508 Chinese patients with myelodysplastic syndrome and comparison with those in Western countries. Leukemia 2005; 19: 767-775, doi: 10.1038/sj.leu.2403688.

6. Naqvi K, Jabbour E, Bueso-Ramos C, Pierce S, Borthakur G, Estrov Z, et al. Implications of discrepancy in morphologic diagnosis of myelodysplastic syndrome between referral and tertiary care centers. Blood 2011; 118: 46904693, doi: 10.1182/blood-2011-03-342642.

7. Pavlu J, Emmerson J, Marks AJ, Bain BJ. Idiopathic cytopenia of undetermined significance and the minimal criteria for a diagnosis of myelodysplastic syndrome. Leuk Lymphoma 2011; 52: 515-516, doi: 10.3109/10428194. 2010.545464.

8. Kern W, Haferlach C, Schnittger S, Haferlach T. Clinical utility of multiparameter flow cytometry in the diagnosis of 1013 patients with suspected myelodysplastic syndrome: correlation to cytomorphology, cytogenetics, and clinical 
data. Cancer 2010; 116: 4549-4563, doi: 10.1002/ cncr.25353.

9. Raess PW, van de Geijn GJ, Njo TL, Klop B, Sukhachev D, Wertheim $\mathrm{G}$, et al. Automated screening for myelodysplastic syndromes through analysis of complete blood count and cell population data parameters. Am J Hematol 2014; 89: 369-374, doi: 10.1002/ajh.23643.

10. Nachtkamp K. Clinical presentation of myelodysplastic syndromes. London: Future Medicine Ltd.; 2014.

11. Corey SJ, Minden MD, Barber DL, Kantarjian H, Wang JC, Schimmer AD. Myelodysplastic syndromes: the complexity of stem-cell diseases. Nat Rev Cancer 2007; 7: 118-129, doi: $10.1038 / \mathrm{nrc2047.}$

12. Flores-Figueroa E, Gutierrez-Espindola G, Montesinos JJ, Arana-Trejo RM, Mayani $\mathrm{H}$. In vitro characterization of hematopoietic microenvironment cells from patients with myelodysplastic syndrome. Leuk Res 2002; 26: 677-686, doi: 10.1016/S0145-2126(01)00193-X.

13. Schneider DR, Picker LJ. Myelodysplasia in the acquired immune deficiency syndrome. Am J Clin Pathol 1985; 84: 144-152.

14. Sacchetti B, Funari A, Michienzi S, Di Cesare S, Piersanti S, Saggio I, et al. Self-renewing osteoprogenitors in bone marrow sinusoids can organize a hematopoietic microenvironment. Cell 2007; 131: 324-336, doi: 10.1016/j.cell. 2007.08.025.

15. Zhao JL, Ma C, O'Connell RM, Mehta A, DiLoreto R, Heath $J R$, et al. Conversion of danger signals into cytokine signals by hematopoietic stem and progenitor cells for regulation of stress-induced hematopoiesis. Cell Stem Cell 2014; 14: 445-459, doi: 10.1016/j.stem.2014.01.007.

16. Perry JM, He XC, Sugimura R, Grindley JC, Haug JS, Ding $\mathrm{S}$, et al. Cooperation between both Wnt/\{beta\}-catenin and PTEN/PI3K/Akt signaling promotes primitive hematopoietic stem cell self-renewal and expansion. Genes Dev 2011; 25: 1928-1942, doi: 10.1101/gad.17421911.

17. Kitagawa M, Kurata M, Yamamoto K, Abe S, Suzuki S, Umeda S. Molecular pathology of myelodysplastic syndromes: biology of medullary stromal and hematopoietic cells (review). Mol Med Rep 2011; 4: 591-596.

18. Song LX, Guo J, He Q, Xu F, Yang LP, Li X, et al. [Cytogenetic abnormalities in bone marrow mesenchymal stem cells from patients with myelodysplastic syndrome]. Zhongguo Shi Yan Xue Ye Xue Za Zhi 2011; 19: 372-378.

19. Flores-Figueroa E, Arana-Trejo RM, Gutierrez-Espindola G, Perez-Cabrera A, Mayani H. Mesenchymal stem cells in myelodysplastic syndromes: phenotypic and cytogenetic characterization. Leuk Res 2005; 29: 215-224, doi: 10.1016/ j.leukres.2004.06.011.

20. Blau O, Hofmann WK, Baldus CD, Thiel G, Serbent V, Schumann E, et al. Chromosomal aberrations in bone marrow mesenchymal stroma cells from patients with myelodysplastic syndrome and acute myeloblastic leukemia. Exp Hematol 2007; 35: 221-229, doi: 10.1016/ j.exphem.2006.10.012.

21. Mallo M, Cervera J, Schanz J, Such E, Garcia-Manero G, Luno E, et al. Impact of adjunct cytogenetic abnormalities for prognostic stratification in patients with myelodysplastic syndrome and deletion 5q. Leukemia 2011; 25: 110-120, doi: 10.1038/leu.2010.231.

22. Jung SW, Lee SY, Jekarl DW, Kim M, Lim J, Kim Y, et al.
Cytogenetic characteristics and prognosis analysis in 231 myelodysplastic syndrome patients from a single institution. Leuk Res 2011; 35: 735-740, doi: 10.1016/j.leukres. 2010.11.009.

23. Senent L, Arenillas L, Luno E, Ruiz JC, Sanz G, Florensa L. Reproducibility of the World Health Organization 2008 criteria for myelodysplastic syndromes. Haematologica 2013; 98: 568-575, doi: 10.3324/haematol.2012.071449.

24. Nwajei $\mathrm{F}$, Konopleva M. The bone marrow microenvironment as niche retreats for hematopoietic and leukemic stem cells. Adv Hematol 2013; 2013: 953982, doi: 10.1155/2013/ 953982.

25. Toth ZE, Leker RR, Shahar T, Pastorino S, Szalayova I, Asemenew B, et al. The combination of granulocyte colonystimulating factor and stem cell factor significantly increases the number of bone marrow-derived endothelial cells in brains of mice following cerebral ischemia. Blood 2008; 111: 5544-5552, doi: 10.1182/blood-2007-10-119073.

26. Zvetkova E, Fuchs D, Katzarova E, Bakalska M, Svetoslavova M, Nikolov B. Neopterin acting as a bone marrow stem cell factor on early common haematopoietic (myeloid) and stromal (dendritic, CD34 +) cell progenitors in vitro. Pteridines 2001; 12: 135-139, doi: 10.1515/pteridines. 2001.12.4.135.

27. Dar A, Schajnovitz A, Lapid K, Kalinkovich A, Itkin T, Ludin $A$, et al. Rapid mobilization of hematopoietic progenitors by AMD3100 and catecholamines is mediated by CXCR4dependent SDF-1 release from bone marrow stromal cells. Leukemia 2011; 25: 1286-1296, doi: 10.1038/leu.2011.62.

28. Dar A, Kollet O, Lapidot T. Mutual, reciprocal SDF-1/CXCR4 interactions between hematopoietic and bone marrow stromal cells regulate human stem cell migration and development in NOD/SCID chimeric mice. Exp Hematol 2006; 34: 967-975, doi: 10.1016/j.exphem.2006.04.002.

29. Zhang Y, Zhao H, Zhao D, Sun L, Zhi Y, Wu X, et al. SDF-1/ CXCR4 axis in myelodysplastic syndromes: correlation with angiogenesis and apoptosis. Leuk Res 2012; 36: 281-286, doi: 10.1016/j.leukres.2011.06.017.

30. Kondo A, Yamashita T, Tamura H, Zhao W, Tsuji T, Shimizu $\mathrm{M}$, et al. Interferon-gamma and tumor necrosis factor-alpha induce an immunoinhibitory molecule, $\mathrm{B} 7-\mathrm{H} 1$, via nuclear factor-kappaB activation in blasts in myelodysplastic syndromes. Blood 2010; 116: 1124-1131, doi: 10.1182/blood2009-12-255125.

31. Cachaço AS, Carvalho T, Santos AC, Igreja C, Fragoso R, Osório $\mathrm{C}$, et al. TNF- $\alpha$ regulates the effects of irradiation in the mouse bone marrow microenvironment. PloS one 2010; 5: e8980, doi: 10.1371/journal.pone.0008980.

32. Cortez M, Carmo LS, Rogero MM, Borelli P, Fock RA. A high-fat diet increases IL-1, IL-6, and TNF-alpha production by increasing NF-kappaB and attenuating PPAR-gamma expression in bone marrow mesenchymal stem cells. Inflammation 2013; 36: 379-386, doi: 10.1007/s10753-0129557-z.

33. Blau O, Baldus CD, Hofmann WK, Thiel G, Nolte F, Burmeister $\mathrm{T}$, et al. Mesenchymal stromal cells of myelodysplastic syndrome and acute myeloid leukemia patients have distinct genetic abnormalities compared with leukemic blasts. Blood 2011; 118: 5583-5592, doi: 10.1182/blood2011-03-343467.

34. Pinheiro RF, Serio FM, Silva MR, Briones MR, Chauffaille 
ML. Association of loss of heterozygosity with cytogenetic abnormalities in acute myeloid leukemia and myelodysplastic syndrome. Braz J Med Biol Res 2008; 41: 610-614, doi: 10.1590/S0100-879X2008000700010.

35. Lopez-Villar O, Garcia JL, Sanchez-Guijo FM, Robledo C,
Villaron EM, Hernandez-Campo $\mathrm{P}$, et al. Both expanded and uncultured mesenchymal stem cells from MDS patients are genomically abnormal, showing a specific genetic profile for the 5q- syndrome. Leukemia 2009; 23: 664-672, doi: 10.1038/leu.2008.361. 